\title{
Numerical Simulation of the Spectral Discretization of Electromagnetic Field Equations
}

\section{Fubiao Lin}

Guizhou University of Finance and Economics, Guiyang, P. R. China

E-mail: linfubiao0851@163.com

Keywords: Electromagnetic field equation, Energy, plane wave basis, Spectral, SCF

\begin{abstract}
: in this paper we use pseudo potentials and plane wave basis to compute the total energy, charge density and electronic structure electromagnetic field equations. Numerical simulation method is an available method to analyze complex flow field.
\end{abstract}

\section{Introduction}

Hartree-Fock theory can be viewed as the real starting point of quantum chemistry. The core physical essence of Hartree-Fock, is the single electron approximation. On base of this approximation, the chemists produce the very common and fundamental chemical picture so widely used in chemistry: the molecular orbital's and the electrons reside on them. The electromagnetic field equation equations that models the behavior of a quantum molecular system deals with state function $\psi\left(x_{-}\right)$,where $\underset{-}{x}$ denotes the position of the particles (nuclei and electrons) hence is a variable that lives in $\mathbb{R}^{3 K}$ where $K$ is the number of particles. The system is too large to be directly tractable by numerical simulation. The quantum chemists have thus introduced a series of simplified models. Combes et al. ${ }^{[1,2]}$ presented to separate the electron and the nuclei so as to consider a system in which only the $N$ electrons of the molecule move and the nuclei are fixed in $\bar{x}_{j}$.For each configuration $\left(\bar{x}_{1}, \cdots, \bar{x}_{m}\right)$ of the $m$ nuclei a complex electronic wave function $\Phi\left(x_{1}, \cdots, x_{N}\right) \in \mathbb{C}$, $x_{i} \in \mathbb{R}^{3}, i=1, \cdots, N$ is sought after that minimizes the energy of the system. Another method is therefore introduced by considering that state function is a $N$ dimensional determinant of simple function of $\mathbb{R}^{3}$ called Slater determinant: $\Phi\left(r_{1}, \cdots, r_{N}\right)=\frac{1}{\sqrt{N !}} \operatorname{det}\left(\Phi_{i}\left(r_{j}\right)\right)$, where $\Phi_{i}, i=1, \cdots, N$ are now functions of one variable in chosen orthogonal respect to the canonical scalar product $\langle\cdot, \cdot\rangle$ on $L^{2}\left(\mathbb{R}^{3}\right)$

Let us denote by $M$ the subset of $L^{2}\left(\mathbb{R}^{3}\right)^{N}$ defined by

$$
M=\left\{\Phi=\left(\phi_{1}, \cdots, \phi_{N}\right)^{T} \in\left(L^{2}\left(\mathbb{R}^{3}\right)\right)^{N} \mid \int_{\mathbb{R}^{3}} \phi_{i} \phi_{j}=\delta_{i, j}\right\},
$$

$N$ being the number of valence electron pairs in the simulation cell, and we define the HartreeFock energy as(see e.g. [3]):

$$
\begin{gathered}
E^{H F}(\Phi)=\sum_{i=1}^{N} \int_{\mathbb{R}^{3}}\left(\left|\nabla \phi_{i}\right|^{2}+V\left|\phi_{i}\right|^{2}\right)+\frac{1}{2} \iint_{\mathbb{R}^{3} \times \mathbb{R}^{3}} \frac{\rho_{\Phi}(x) \rho_{\Phi}(y)}{|x-y|} d x d y \\
-\frac{1}{2} \iint_{\mathbb{R}^{3} \times \mathbb{R}^{3}} \frac{\left|\rho_{\Phi}(x, y)\right|^{2}}{|x-y|} d x d y
\end{gathered}
$$


where the density matrix $\rho_{\Phi}(x, y)$ the electronic density $\rho_{\Phi}(x)$ and the potential $V$ are given by the formulates:

$$
\rho_{\Phi}(x, y)=\sum_{i=1}^{N} \phi_{i}(x) \overline{\phi_{j}(y)}, \quad \rho_{\Phi}(x)=\rho_{\Phi}(x, x), V(x)=-\sum_{j=1}^{m} \frac{Z_{j}}{\left|x-\bar{x}_{j}\right|},
$$

here, we have denoted by $Z_{j}>0$ the charge of the j-th nuclei.

The main difficulties for numerical approximations of the ground state solutions of electromagnetic field equation include:on the one hand, the associate energy functional is not convex with respect to $\rho$. The existence of a minimizer of (1) is known for the models under appropriate physically relevant conditions (see $[4,5,6]$ ). In contrast, nothing is known about the uniqueness of a solution of density $\rho$ or equivalently $\phi \geq 0$, which will bring serious difficulty for numerical analysis. In most cases, we need to apply some sophisticated arguments to deal with the nonconvex effects when we carry out the numerical analysis. On the other hand, due to the existing of the nonlinear terms, there are several difficulties in obtaining convergence and a priori error estimate of the numerical approximate solutions. In particular, the nonlocal convolution integration part will induce many troubles for numerical analysis. At last, efficient numerical algorithms for solving nonlinear eigenvalue problems are lacked. Special numerical strategies for solving the corresponding nonlinear eigenvalue problem of (1) should be carefully chosen or designed. In most applications, a number of eigenpairs are desired and the worst thing is that the self-consistent iteration is not so easy to converge and it often takes several tens of steps.

The Hartree-Fock equations have gained a surge of attention in both analytical and numerical aspects. For neutral atoms and molecules and positive ions and radicals, Lieb, Simon ${ }^{[4]}$, and Lions ${ }^{[5]}$ have proved the existence of solutions of the Hartree-Fock equations which minimize the HartreeFock energy. In ${ }^{[3]}$, Maday \&Turinici presented a posteriori error analysis for the approximation of the Hartree-Fock equations. Flad et al. ${ }^{[7]}$ presented the asymptotic regularity of solutions of HartreeFock equations for Coulomb systems. In order to deal with singular Coulomb potentials, Fock operators are discussed within the calculus of pseudo-differential operators on conical manifolds. Within the local density approximation, Cances et.al ${ }^{[8]}$ have provide some priori error estimates for the spectral and pseudo spectral discretizations for some convex energy functional model.

Some other work has been focused on using numerical methods to simulate the behavior of the solutions of Hartree-Fock equations. In ${ }^{[9]}$, Cances \& Le Bris have presented a two algorithms, namely the Roothaan and the level-shifting algorithms to solve the Hartree-Fock equations. Flad et al. ${ }^{[10]}$ discussed the efficient rank structured tensor approximation method for electronic structure calculations. Because of the absence of many-electron cusps in these models, they restrict themselves to the electron-nuclear cusp, where a single electron approaches a nucleus. In ${ }^{[11]}$, a trust region technique is introduced as a way to restrict the update of the wave functions within a small neighborhood of an approximate solution at which the gradient of the total energy of Kohn-Sham equation.

In this paper we use pseudo potentials and plane wave basis to compute the total energy, charge density and electronic structure electromagnetic field equations. Numerical simulation method is an available method to analyze complex flow field.

The remainder of this paper is arranged as follows: In the coming section we briefly introduce the functional setting used on the formulation. In Section 3 we introduce Fourier approximation of the electromagnetic field equation. Finally, we report several numerical applications of electronic structure calculations based on spectral descretization.

\section{Preliminaries}

In this context, the simulation domain referred to as the

supercell, is the unit cell of some periodic lattice of $\mathbb{R}^{3}$. In the Hartree-Fock equations, periodic boundary conditions are imposed to the density. We denote by $\Gamma$ the simulation cell, by $R$ the 
periodic lattice, and by $R^{*}$ the dual lattice. For simplicity, we assume that $\Gamma=[0, L)^{3},(L>0)$, in which case $R$ is the cubic lattice $L \mathbb{Z}^{3}$, and $R^{*}=\frac{2 \pi}{L} \mathbb{Z}^{3}$. Our argument can be easily extended to the general case, for $k \in R^{*}$, we also denote by $e_{k}(x)=|\Gamma|^{-\frac{1}{2}} e^{i k \cdot x}$ the planewave with wavevector $k$. The family $\left(e_{k}\right)_{k \in R}^{*}$ forms an orthonormal basis of $L_{\#}^{2}(\Gamma, \mathbb{C}):=\left\{u \in L_{\text {loc }}^{2}\left(\mathbb{R}^{3}, \mathbb{C}\right) \mid u R\right.$ - periodic $\}$

And for all $u \in L_{\#}^{2}(\Gamma, \mathbb{C})$,

$$
u(x)=\sum_{k \in R^{*}} \hat{u_{k}} e_{k}(x) \text { with } \hat{u_{k}}=\left(e_{k}, u\right)_{L_{\#}^{2}}=\left|\Gamma^{-1 / 2}\right| \int_{\Gamma} u(x) e^{-i k \cdot x} .
$$

And we also introduce the Sobolev spaces of real valued

$R$ - Periodic functions

$$
H_{\#}^{s}(\Gamma):=\left\{u(x)=\left.\sum_{k \in R^{*}} \hat{u_{k}} e_{k}(x)\left|\sum_{k \in R^{*}}\left(1+|k|^{2}\right)^{s}\right| \hat{u_{k}}\right|^{2}<\infty \quad \text { and } \forall k, \hat{u_{-k}}=\hat{u}_{k}^{*}\right\},
$$

$s \in \mathbb{R}$ (where $a^{*}$ denotes the complex conjugate of the complex number a), endowed with the inner products

$$
(u, v)_{H_{\#}^{s}}=\sum_{k \in R^{*}}\left(1+|k|^{2}\right)^{s} \hat{u_{k}^{*}} \hat{v}_{k}
$$

For $N_{c} \in \mathbb{N}$, we denote by

$$
V_{N_{c}}=\left\{\sum_{k \in R^{*} \mid k \| \leqslant \frac{2 \pi}{L} N_{c}} \hat{u_{k}} e_{k} \mid \forall k, c_{-k}=c_{k}^{*}\right\}
$$
is

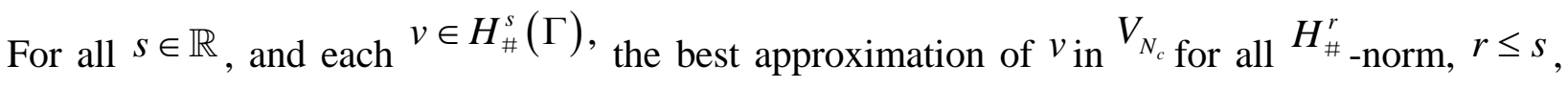

$$
\Pi_{N_{c}} v=\sum_{k \in R^{*}|| k \mid \leq \frac{2 \pi}{L} N_{c}} \hat{u}_{k} e_{k} .
$$

For all real numbers $r$ and $s$ with $r \leq s$, we know the following estimates hold [21]:

$$
\left\|v-\Pi_{N_{c}} v\right\|_{H_{\#}^{s}}=\min _{v_{N_{c}} \in V_{N_{c}}}\left\|v-v_{N_{c}}\right\|_{H_{\#}^{s}} \leq\left(\frac{L}{2 \pi}\right) N_{c}^{-(s-r)}\|v\|_{H_{\#}^{s}} .
$$

\section{Fourier approximation of the Hartree-Fock model}

Let us study the following minimization problem

$$
I^{H F}=\inf \left\{E^{H F}(\Phi), \Phi \in M\right\}
$$

where $M=\left\{\Phi=\left(\phi_{1}, \cdots, \phi_{N}\right)^{T} \in\left(H_{\#}^{1}(\Gamma)\right)^{N} \mid \int_{\Gamma} \phi_{i} \phi_{j}=\delta_{i, j}\right\}, N$ being the number of valence electron pairs in the simulation cell, and where

$$
E^{H F}(\Phi)=\sum_{i=1}^{N} \int_{\Gamma}\left(\left|\nabla \phi_{i}\right|^{2}+V\left|\phi_{i}\right|^{2}\right)+\frac{1}{2} \iint_{\Gamma \times \Gamma} \frac{\rho_{\Phi}(x) \rho_{\Phi}(y)}{|x-y|} d x d y-\frac{1}{2} \iint_{\Gamma \times \Gamma} \frac{\left|\rho_{\Phi}(x, y)\right|^{2}}{|x-y|} d x d y .
$$


The basic result of uniqueness of the density solution is still an open problem. Under the hypothesis $\sum_{j=1}^{m} Z_{j}>N-1$, it has been proven in ${ }^{[5]}$ that a minimum of the problem (3) exists. We will assume in all that follows that (3.3) is true. Troullier \& Martins [22] have proved a popular class of pseudoptentials for which the Fourier coefficients $\hat{V}_{k}$ decay as $|k|^{-5}$ with $m=5$. In all what follows, we will assume that

$$
\exists m>3, C>0 \text { s.t. } \forall k \in R^{*},\left|\hat{V}_{k}\right| \leq C|k|^{-m}
$$

Let $\Phi^{0}=\left(\phi_{1}^{0}, \cdots, \phi_{N}^{0}\right)^{T} \in M$ be a minimum of (4). The regularity assumption on $\mathrm{V}$ allow to state the minimizer $\Phi^{0}$ is in $\left[H_{\#}^{3}(\Gamma)\right]^{N}$ and even in $\left[H_{\#}^{m+1 / 2-\varepsilon}(\Gamma)\right]^{N}$ for all $\varepsilon>0$.

Let us introduce the Hartree-Fock Hamiltonian (seee.g. [3])

$$
H_{\Phi}^{H F}(\psi)(x)=\left(-\Delta+V+\rho_{\Phi} * \frac{1}{|x|}\right) \psi(x)-\int_{\Gamma} \frac{\rho_{\Phi}(x, y) \psi(y)}{|x-y|} d y
$$

where $*$ is the convolution product

$$
(f * g)(x)=\int_{\Gamma} f(x-y) g(y) d y .
$$

We notice that $E^{H F^{\prime}}\left(\Phi^{0}\right)=2 H_{\Phi^{0}}^{H F} \Phi^{0}$ in $\left(H_{\#}^{-1}(\Gamma)\right)^{N}$ and thus the Euler equations associated with the minimization problem (4) read:

$$
H_{\Phi^{0}}^{H F} \Phi_{i}^{0}=-\sum_{j=1}^{N} \lambda_{i j}^{0} \phi_{j}^{0}, 1 \leq i \leq N
$$

\section{Results}

In order to evaluate the quality of the error bounds, we have performed numerical tests using the KSSOLV, whose main program allows one to find the total energy, charge density and electronic structure of systems (molecules and periodic solids) within Hartree-Fock equations, using pseudo potentials and a plane wave basis

The SCF errors reported in this paper are the residual norms de_ned as

$$
\text { error }=\|H(X) X-X \Lambda\|_{F},
$$

where $X$ contains the wave functions returned from SCF and $\Lambda=X * H(X) X$.

Table 1. Comparing the timing (in seconds) and accuracy of runing SCF.

\begin{tabular}{c|cc}
\hline system & SCF time & SCF error \\
$\mathrm{NiC}$ & $4.992 \mathrm{e}+001$ & $2.799 \mathrm{e}-005$ \\
$\mathrm{H}_{2} \mathrm{O}$ & $2.975 \mathrm{e}+001$ & $1.539 \mathrm{e}-006$ \\
$\mathrm{CO}_{2}$ & $4.000 \mathrm{e}+001$ & $6.035 \mathrm{e}-006$ \\
$\mathrm{Si}_{2} \mathrm{H}_{4}$ & $3.964 \mathrm{e}+001$ & $3.863 \mathrm{e}-004$ \\
$\mathrm{C}_{2} \mathrm{H}_{6}$ & $4.047 \mathrm{e}+001$ & $4.500 \mathrm{e}-006$ \\
$\mathrm{C}_{12} \mathrm{H}_{26}$ & $4.703 \mathrm{e}+002$ & $1.008 \mathrm{e}-003$
\end{tabular}

The numerical results for $\mathrm{CO} 2$ are plotted in Figures 1, 2. The numerical results for Si2H4 are plotted in Figures 3, 4. In Figure5, 6, we show the issuer face rendering of the charge density rho returned from the SCF function. 


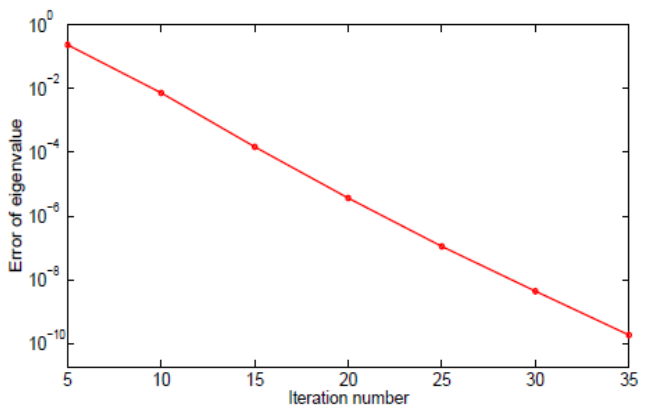

Figure 1. The first smallest eigenvalue in SCF for $\mathrm{CO}_{2}$.

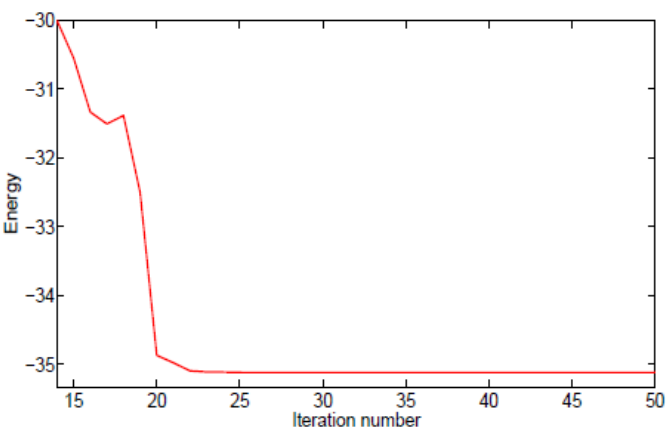

Figure 2. The total energy in SCF for $\mathrm{CO}_{2}$.

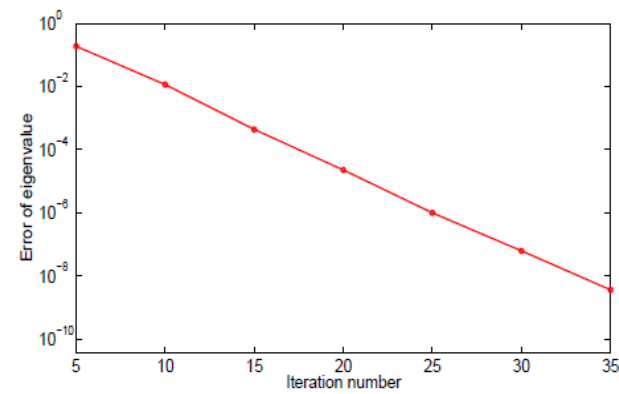

Figure 3. The first smallest eigenvalue in SCF for $\mathrm{Si}_{2} \mathrm{H}_{4}$.

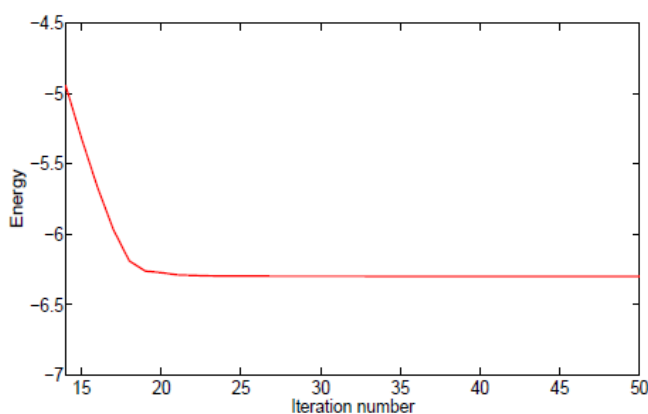

Figure 4. The total energy in SCF for $\mathrm{Si}_{2} \mathrm{H}_{4}$.

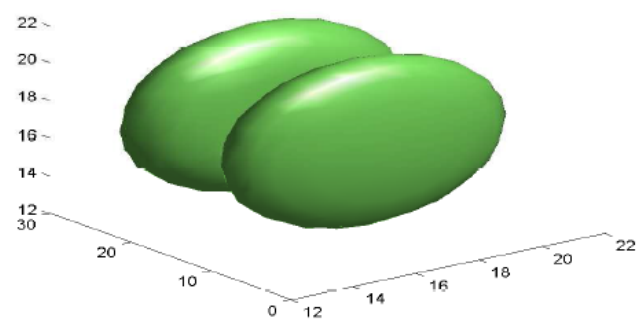

Figure 5. The computed charge density of the $\mathrm{CO}_{2}$ molecule. 


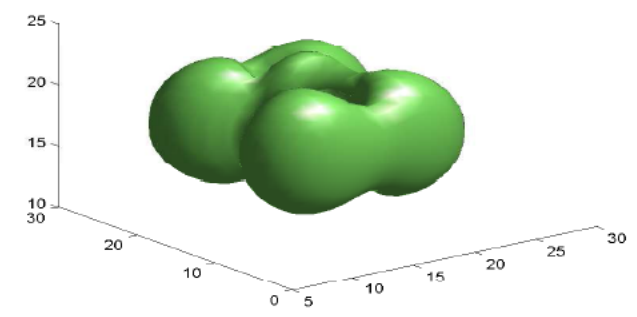

FigurE 6. The computed charge density of the $\mathrm{Si}_{2} \mathrm{H}_{4}$ molecule.

\section{Acknowledgment}

This work partially supported by Natural Science Foundation of Guizhou under Grant (No.[2016] 170 and No.[2017] 150)

\section{References}

[1] J. M. Combers. On the born-oppenheimer approximation. In International Symposium on Mathematical Problems in Theoretical Physics, pages 467-471. Springer, 1975.

[2] J. M. Combers. P. Duclos and R. Seiler. The born-oppenheimer approximation. In Rigorous atomic and molecular physics, pages 185-213. Springer, 1981.

[3] Maday and G. Turinici. Error bars and quadratic ally convergent methods for the numerical simulation of the hartree-fock equations. Numerische Mathematik, 94(4):739-770, 2003.

[4] E. H. Lieb and B. Simon. The thomas-fermi theory of atoms, molecules and solids. Advances in Mathematics, 23(1):22-116, 1977.

[5] P. L. Lions. Solutions of hartree-fock equations for coulomb systems. Communications in Mathematical Physics, 109(1):33-97, 1987.

[6] E. H. Lieb. Thomas-fermi and related theories of atoms and molecules. Reviews of Modern Physics, 53(4):603, 1981.

[7] H. J. Flad, R. Schneider, and B.W. Schulze. Asymptotic regularity of solutions to hartree-fock equations with coulomb potential. Mathematical Methods in the Applied Sciences, 31(18):21722201, 2008.

[8] E. Cances, R. Chakir, and Y. Maday. Numerical analysis of nonlinear eigenvalue problems. Journal of Scientific Computing, 45(1-3):90-117, 2010.

[9] E Cances and C. Le Bris. On the convergence of scf algorithms for the hartree-fock equations. ESAIM: Mathematical Modeling and Numerical Analysis, 34(04):749-774, 2000.

[10] H. J. Flad, W. Hackbusch, and R. Schneider. Best n-term approximation in electronic structure calculations.ii. jastrow factors. ESAIM: Mathematical Modeling and Numerical Analysis, 41(02):261-279, 2007.

[11] C. Yang, J. C. Meza, and L.W. Wang. A trust region direct constrained minimization algorithm for the kohnsham equation. SIAM Journalon Scientific Computing, 29(5):1854-1875, 2007. 